\title{
The Adiabatic Matching Section Solution for the Source Injector*
}

\author{
L. AHLE ${ }^{a, 1}$, D. P. GROTE ${ }^{a}$, E. HALAXA ${ }^{a}$ \\ E. HENESTROZA', J. W. KWAN ${ }^{\text {b }}$, S. A. MACLAREN ${ }^{\mathrm{b}}$ \\ ${ }^{a}$ Lawrence Livermore National Laboratory, Livermore, CA, 94551 USA \\ ${ }^{b}$ Lawrence Berkeley National Laboratory, CA, Berkeley 94720 USA
}

Typical designs for a Heavy Ion Fusion Power Plant require the source injector to deliver 100 beams, packed into an array with a spacing of $7 \mathrm{~cm}$. When designing source injectors using a single large aperture source for each beam, the emitter surfaces are packed into an array with a spacing of $30 \mathrm{~cm}$. Thus, the matching section of the source injector must not only prepare the beam for transport in a FODO lattice, but also funnel the beams together. This can be accomplished by an ESQ matching section in which each beam travels on average at a slight angle to the axis of the quadrupoles and uses the focussing effect of the FODO lattice to maintain the angle. At the end of the matching section, doublet steering is used to bring the beams parallel to each other for injection into the main accelerator. A specific solution of this type for an 84-beam source injector is presented. PACS: 41.75.Ak,41.85.Ar, 41.85.Ja

Keywords: beam injection, beam transport, heavy ion fusion

*This work was performed under the auspices of the U.S. Department of Energy by University of California Lawrence Livermore National Laboratory under contract No. W-7405-Eng-48 and by University of California Lawrence Berkeley Laboratory under contract DE-AC03-76SF00098.

${ }^{1}$ Corresponding Author, L-645, LLNL, P.O. Box 808, Livermore, CA 94551; Tel.: 925422-1621; Fax: 925-422-1767; E-mail: ahle1@1lnl.gov 


\section{Introduction}

In a typical design for a heavy ion inertial fusion power plant, the main accelerator accepts an array of approximately 100 beams with a spacing of $7 \mathrm{~cm}$ between beams. Each beam has a radius of $1 \mathrm{~cm}$, a current of $0.5 \mathrm{~A}$ and a kinetic energy of $1.6 \mathrm{MeV}$ [1]. But due to transport limitations from space charge and voltage breakdown, producing each of these 100 beams from a single source results in a source gun delivering an array with a $30 \mathrm{~cm}$ spacing [2]. The beams leaving the source gun have a radius of $3 \mathrm{~cm}$ but the radius of the emitter surface is $7 \mathrm{~cm}$ which, together with the size of the anode, determines the spacing. Thus, there must be some sort of matching section between the injector and the accelerator that funnels the beams down to the smaller array size. One possible solution for this matching section is the approach we have named the Adiabatic Matching Section.

\section{The Adiabatic Concept}

The idea behind the Adiabatic Matching Section is simple. Given that a beam tends to be confined within a transport channel if the channel varies slowly enough, one could use a matching section that slowly varies the array size from the large output of the injector to the small input for the accelerator. But varying the size slowly means a long pathlength, so to minimize cost and the longitudinal expansion of the beam, it is desirable to minimize the length of the matching section.

In the Adiabatic Matching Section, a beam will see a channel as shown in figure 1. The axis of each quadrupole is parallel but offset to its downstream and upstream neighbors. The beam will tend to follow a path at an angle to the quadrupole axis. At the beginning of the accelerator centroid offsets of $1 \mathrm{~cm}$ relative to the quadrupole axis would be tolerable before the beam leaves the good field region of the ESQ. Figure 2 plots the centroid positions at the beginning and end of each quadrupole relative to the quadrupole 
axis for the offset quadrupole channel described above. The centroid positions are determined from solving the equations of motion of a single charged particle in the lattice. The offset, $d$, between quadrupoles is $2 \mathrm{~cm}$ and the results for two different initial centroid trajectories are shown. If the beam enter the first quadrupole on its axis, then a betatron oscillation occurs with an amplitude of about $3 \mathrm{~cm}$, as shown by the square points in figure 2. However, if the beam is initially aimed down the channel, then the centroid positions stay within $1 \mathrm{~cm}$ of the quadrupole axis. The initial trajectory of the beam to minimize the oscillations, $x=0.667 \mathrm{~cm}$ and $x^{\prime}=111 \mathrm{mr}$, is simply the straight line path that crosses all the quadrupole axis at their respective mid-planes.

It is quite feasible for the source gun to aim the beams to enter the matching section with the appropriate trajectories, but the beams must leave the matching section traveling parallel to each other. Thus, the beams must be steered, but given the space constraints in the lattice of the ESQ it is desirable not to insert extra elements to steer the beam. Another method of steering is to change the offset of the quadrupoles, but in order to minimize the betatron motion of the beam, this is most effectively done by changing the offset in pairs, i.e., doublet steering. Figure 3 shows the result for same lattice as in figure 2 but with doublet steering spread out over the first 10 quadrupoles, that is, if $d_{n}$ is the offset for the $n$th quadrupole, then $d_{1}$ and $d_{2}$ are $0.2^{*} d, d_{3}$ and $d_{4}$ are $0.4^{*} d, d_{5}$ and $d_{6}$ are $0.6^{*} d, d_{7}$ and $d_{8}$ are $0.8 * d$, and $d_{9}$ through $d_{n}$ are $d$. Again, in this case $\mathrm{d}=2 \mathrm{~cm}$ and the beam enters the first quadrupole on axis. The plots shows quite clearly the reduction in the largest centroid offset from $3 \mathrm{~cm}$ to $1 \mathrm{~cm}$, demonstrating doublet steering provides an adequate method to bring the beam trajectories parallel to each other in an Adiabatic Matching Section.

\section{Designing an 84 Beam Matching Section}

The design for the quadrupole arrays of an 84 beam matching section is based on a design for the Elise heavy ion induction accelerator [3]. The 84 beam array configuration is 
simply a $10 \times 10$ grid with a 2 x 2 grid removed from each corner. The quadrupoles are constructed from two plates from which cylinders extend from both sides to serve as electrodes for the ESQ's. The plates are $2 \mathrm{~cm}$ thick with the negative electrodes extending from one plate and the positive from the other. The gap between the electrodes and the opposite plate is determined by the minimum allowed before voltage breakdown.

For quadrupoles formed by cylindrical electrodes, the next highest allowed pole beyond the quadrupole, the dodecapole, can be eliminated by the setting the electrode radius to $8 / 7$ the bore radius[4]. Given the spacing between beams and the $8 / 7$ rule, this determines the bore radius of the matching section for the close packed quadrupole array of the matching section. In a close pack array, electrodes are shared by more than one quadrupole. In fact, all the electrodes on the inside of the configuration are shared by four quadrupoles. Since the spacing shrinks throughout the matching section, the bore radius shrinks from $10 \mathrm{~cm}$ at the gun end, to $2.3 \mathrm{~cm}$ at the accelerator end.

In an effort to minimize the length of the matching section, the voltage on the electrode is set to as large as possible. The voltage, however, is limited by breakdown considerations. For distances larger than $1 \mathrm{~cm}$, the design limitation for voltage breakdown between two metal surfaces in vacuum,

$$
V_{b}=10^{6} z^{1 / 2}
$$

where $V_{b}$ is in volts and $z$ is in meters, is used [4]. This relation has a safety factor of about two. For the ESQ, the distance that determines the voltage breakdown limit is the distance between the positive and negative electrode surfaces. Inserting this distance in equation 1, the following relation for the quadrupole voltage is obtained,

$$
V_{q}=10^{6}(b(15 \sqrt{2}-16) / 7)^{1 / 2}
$$

where $V_{q}$ is the voltage between the positive and negative electrodes of the quadrupole. Note that the quadrupole voltage scales as the bore radius to the $1 / 2$ power, meaning that as the 
aperture of the quadrupole gets bigger, the gradient get smaller. Equations 1 and 2 then sets the longitudinal spacing between electrodes and the opposite plate, as mentioned above.

In order to keep the envelope matched throughout the matching section, the phase advance, $\sigma_{0}$, of the lattice is maintained as a constant $76^{\circ}$ in this design. Since the gradient increases along the matching section, the length of the quadrupole must be shortened in order to maintain a constant phase advance. To determine this length, the thick lens approximation is used, namely

$$
L=b\left|\frac{2\left(1-\cos \sigma_{0}\right)}{\mid \eta^{2}\left(1-\frac{2 \eta}{3}\right)\left(\frac{V_{q}}{2 V}\right)^{2}}\right|^{\eta^{1 / 4}}-s
$$

where $\mathrm{V}$ is the gun voltage, $1.6 \mathrm{MV}$ in this case, and

$$
\eta=\frac{L}{L+s}
$$

is the occupancy of the lattice. At the gun end of the lattice where $b=10 \mathrm{~cm}$, the effective quadrupole length is $40 \mathrm{~cm}$, the gap, $s$, is $9.1 \mathrm{~cm}$, and the voltage between electrodes is 270 $\mathrm{kV}$, while at the injector end, where $b=2.3 \mathrm{~cm}, l$ is $13.4 \mathrm{~cm}, s$ is $3.7 \mathrm{~cm}$, and $V q$ is $130 \mathrm{kV}$.

Knowing the output of the matching section requires the beams on axis and parallel to each other, the offsets of the matching section were determined by starting from the accelerator end and following the beam centroids backward through the lattice. The beams were steered until the beam furthest away from the center of the array is traveling at angle of $111 \mathrm{mr}$ relative to the quadrupole axis. This trajectory was maintained until the required spacing at the gun end of the matching section was obtained. Since the gun is aiming the beams together and only requires the $30 \mathrm{~cm}$ spacing at the emitter surface end, the minimum spacing at the end of the gun is about $28 \mathrm{~cm}$, assuming an angle of $111 \mathrm{mr}$ for the beam furthest away from the center. It should be noted that for a given quadrupole array the offsets and the angle grows the farther away the beam is from the center. Also, the doublet steering was spread out over 20 doublet pairs, i.e. 5\% steps. This slow rate of steering was 
needed to minimize oscillations in the system. The offsets also had to be scaled up in order to maintain the angle, since both $L$ and $s$ are larger at the gun end. A design with an angle of $140 \mathrm{mr}$ was also considered, but the centroid offsets were found to be too large.

The last part of the design is altering the first four quadrupoles in order to match the envelope from the source gun to the quadrupole lattice. This done by shortening the first quadrupole to $2 / 3$ of its original value and then running an envelope solver which optimizes the voltages. A matched beam can be achieved throughout the entire matching section because the phase advance remains the same even though the average radius does shrink because the quadrupole gradient increases. This change is taken into account by defining the match criteria for solver to be

$$
\left\lfloor\begin{array}{l}
\left.x_{\text {env }}\left(z_{2}\right)\right\rceil \\
\left.y_{\text {env }}\left(z_{2}\right)\right\rfloor
\end{array}\right\rfloor=\left[\begin{array}{l}
\left.x_{\text {env }}\left(z_{1}\right)\right\rceil \\
y_{\text {env }}\left(z_{1}\right)
\end{array}\right\rfloor\left(1+\left(\left(g_{6} l_{6}-g_{8} l_{8}\right) / g_{6} l_{6}\right)\right)
$$

and

$$
\left[\begin{array}{l}
\left.x_{\text {env }}\left(z_{1}\right)\right\rceil \\
\left.x_{\text {env }}\left(z_{2}\right)\right\rfloor
\end{array}\right\rfloor=\left\lfloor\begin{array}{l}
\left.y_{\text {env }}\left(z_{1}\right)\right\rceil \\
y_{\text {env }}\left(z_{2}\right)
\end{array}\right\rfloor
$$

where $x_{e n v}$ and $y_{e n v}$ are the $x$ and $y$ envelopes of the beam, $z_{1}$ is the position half way in between the fifth and sixth quadrupole, $z_{2}$ is the position halfway between the seventh and eighth quadrupole, and $g_{n}$ and $l_{n}$ are the gradient and effective length of the $n$th quadrupole. The resulting voltages only vary about $20 \%$ from the nominal value, so there is little change in the trajectories of the beams. Also, since the first quadrupole was shortened, the offset must also be reduced in order to maintain the $111 \mathrm{mr}$ angle. The final result is a lattice that is $14.3 \mathrm{~m}$ long, consisting of 54 quadrupoles that starts with a quadrupole array that is $3 \mathrm{~m}$ x $3 \mathrm{~m}$ and ends with one that is $0.7 \mathrm{~m}$ x $0.7 \mathrm{~m}$. Table 1 list some details for the several of the quadrupoles.

\section{PIC Simulations}


After designing the matching section, a particle-in-cell code, WARP [5], was used to simulate the beam travelling through the matching section in order to investigate beam loss, emittance growth, and phase-space distortion due to field aberrations from the mechanical design of the quadrupole. The results presented are from a 2D simulation in which the beam was initially loaded with full current and a semi-gaussian distribution. Only one beam line of the matching section was simulated, specifically the beam line furthest away from the center of the arrays. Since this has the largest offsets and centroid oscillations, the effects should be largest in this beam line. In doing the envelope matching in the conceptual design phase, no non-linear effects were considered, but in the simulations, the non-linear effects result in an envelope that is not well matched. To truly minimize these effects, the envelope matching must be fine tuned with the non-linear effects taken into account. This was not done for the results presented here. Thus, effects seen in this study are an upper bound on what would be seen in reality.

Some beam loss is seen through the matching section, about $1.5 \%$ by the end. This amount of beam loss is not expected to be a problem in a driver. In studying the emittance, the simulations shows a initial emittance spike as the beam is matched from the source to the lattice. After the beam is matched, though the normalized emittance quickly returns back to the original value, $1.0 \pi$-mm-mr. So no significant emittance growth is seen. Figures 4 and 5 are plots of the phase space of the beam at $17.1 \mathrm{~m}$ downstream of the source, or about $3 \mathrm{~m}$ after the end of the matching section. Figure 4 is a plot of the $\mathrm{x}-\mathrm{x}$ ' phase space, while figure 5 is a plot of the $x-y$ phase space. All the plots show some evidence of low level of halo formation. These plots show no significant phase space distortions due to non-linear effects.

\section{Conclusion}

It is possible to use a ESQ matching section to solve the size mismatch between the source gun and the accelerator in typical designs of a HIF driver. The matching section 
consists of close packed quadrupole arrays that slowly shrink in size until the beam spacing matches that of the accelerator. This type of matching section, called the Adiabatic Matching Section, would be $14 \mathrm{~m}$ long, consisting of 54 quadrupoles. The source gun would need to initially aim the beams together but the matching section would use doublet steering to bring the beam travelling parallel to each other at the end of the matching section. PIC simulation show minimal beam loss, emittance growth, and phase-space distortion due to the beams travelling through this matching section. 


\section{References}

[1] R. O. Bangerter, Nuovo Cimento 106A (11), 1445 (1993).

[2] J. W. Kwan, et. al., Nuovo Cimento 106A (11), 1541 (1993).

[3] L. Reginato and C. Peters, Fusion Engineering and Design, 32-33, 299 (1996).

[4] Ed Lee, et. al., Fusion Engineering and Design, 32-33, 323 (1996).

[5] D. P. Grote, et. al., Fusion Engineering and Design, 32-33, 193 (1996). 


\section{Figures}

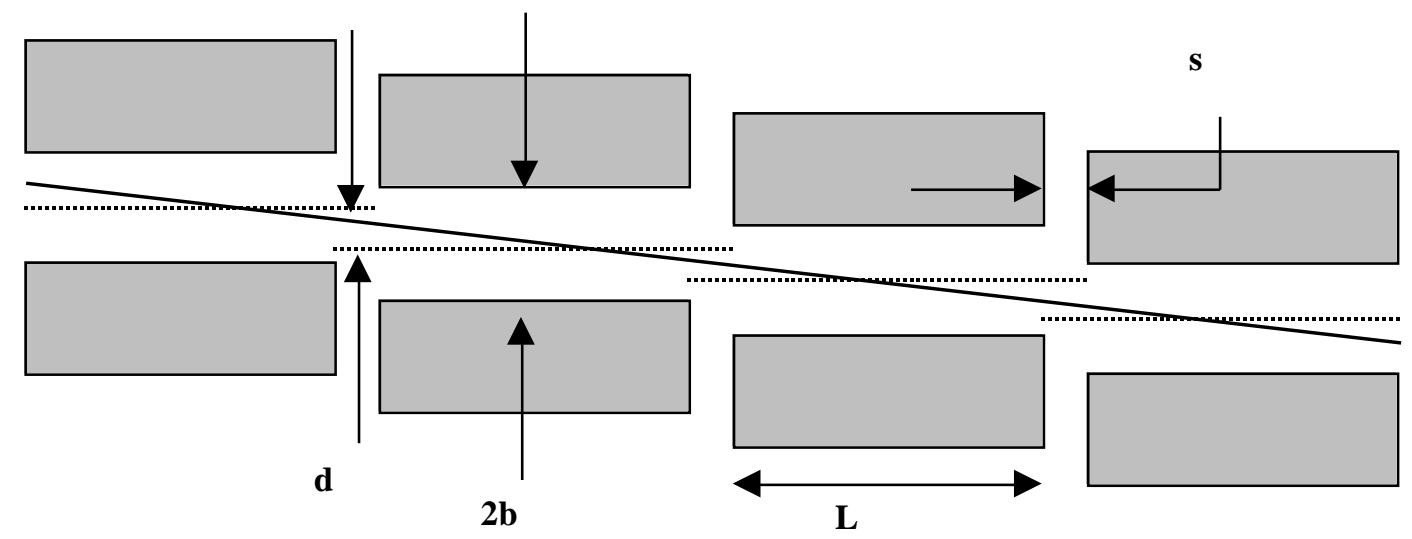

Figure 1: Diagram of offset quadrupole channel present in the Adiabatic Matching Section. The solid line is the approximate beam trajectory. 


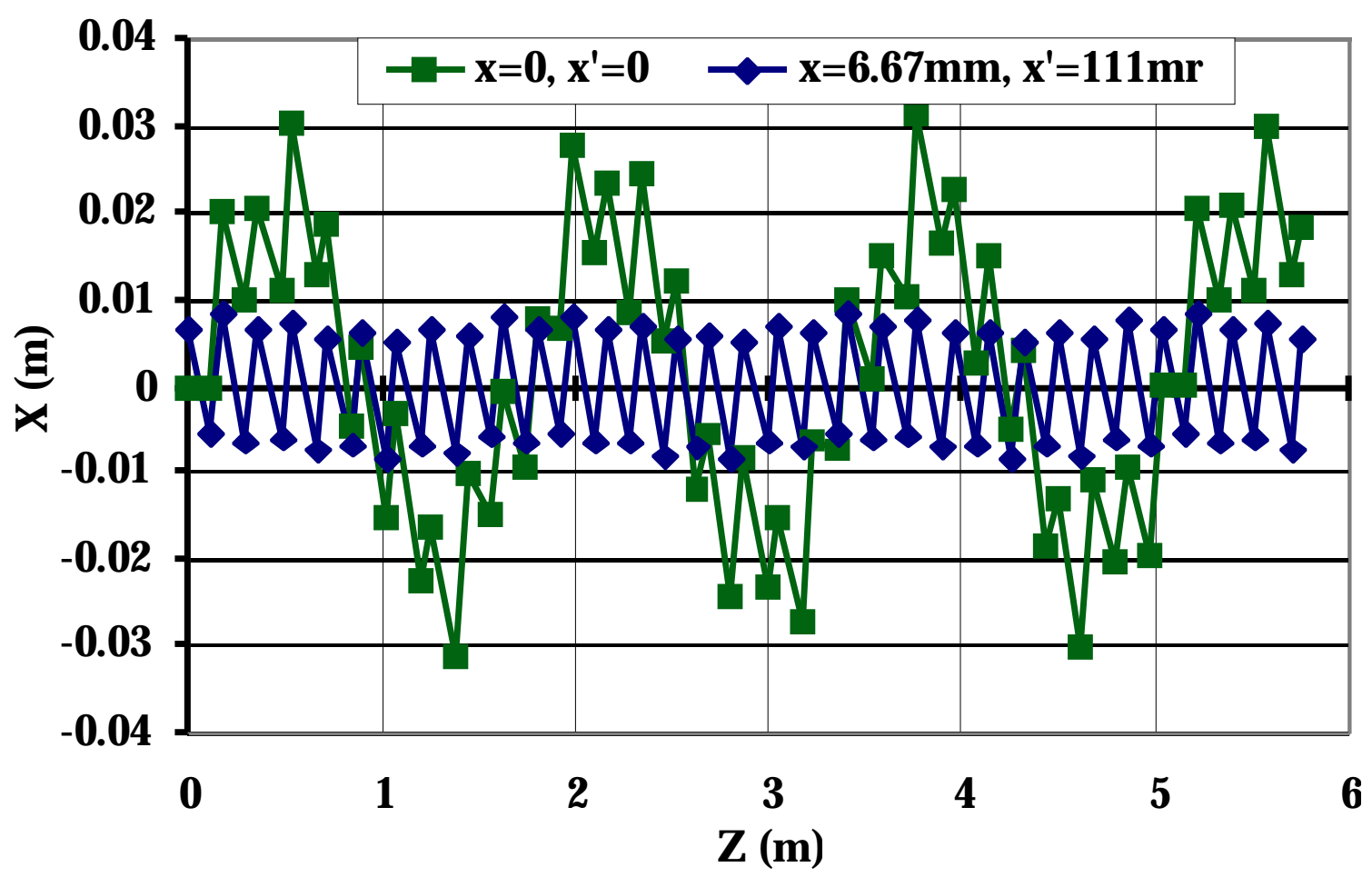

Figure 2: Plots of the beam centroid positions relative to the local quadrupole axis in an offset quadrupole channel with $\mathrm{d}=2.0 \mathrm{~cm}$. The two plots are for two different initial centroid trajectories. 


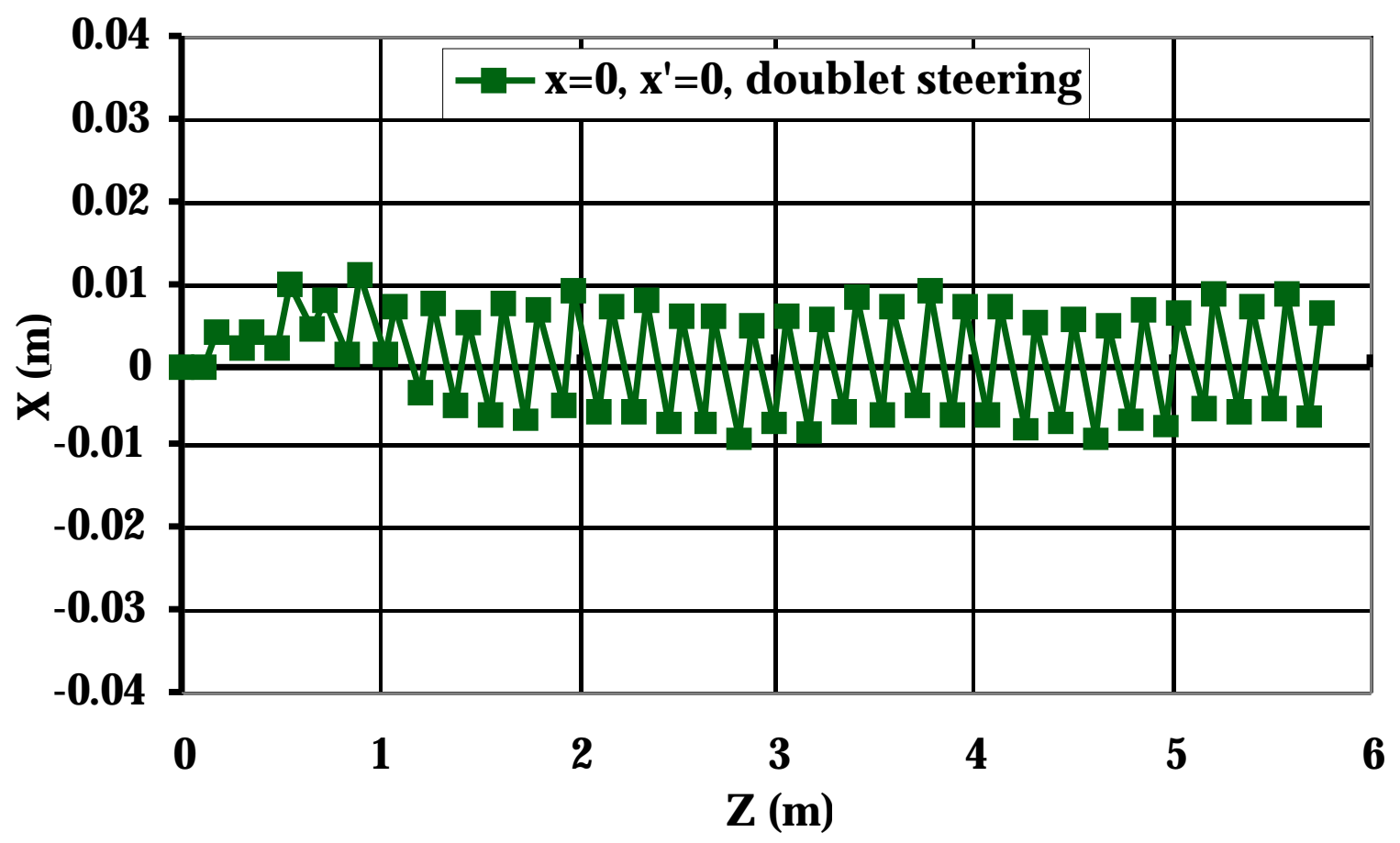

Figure 3: Plot of centroid positions relative to local quadrupole axis for case with doublet steering. 


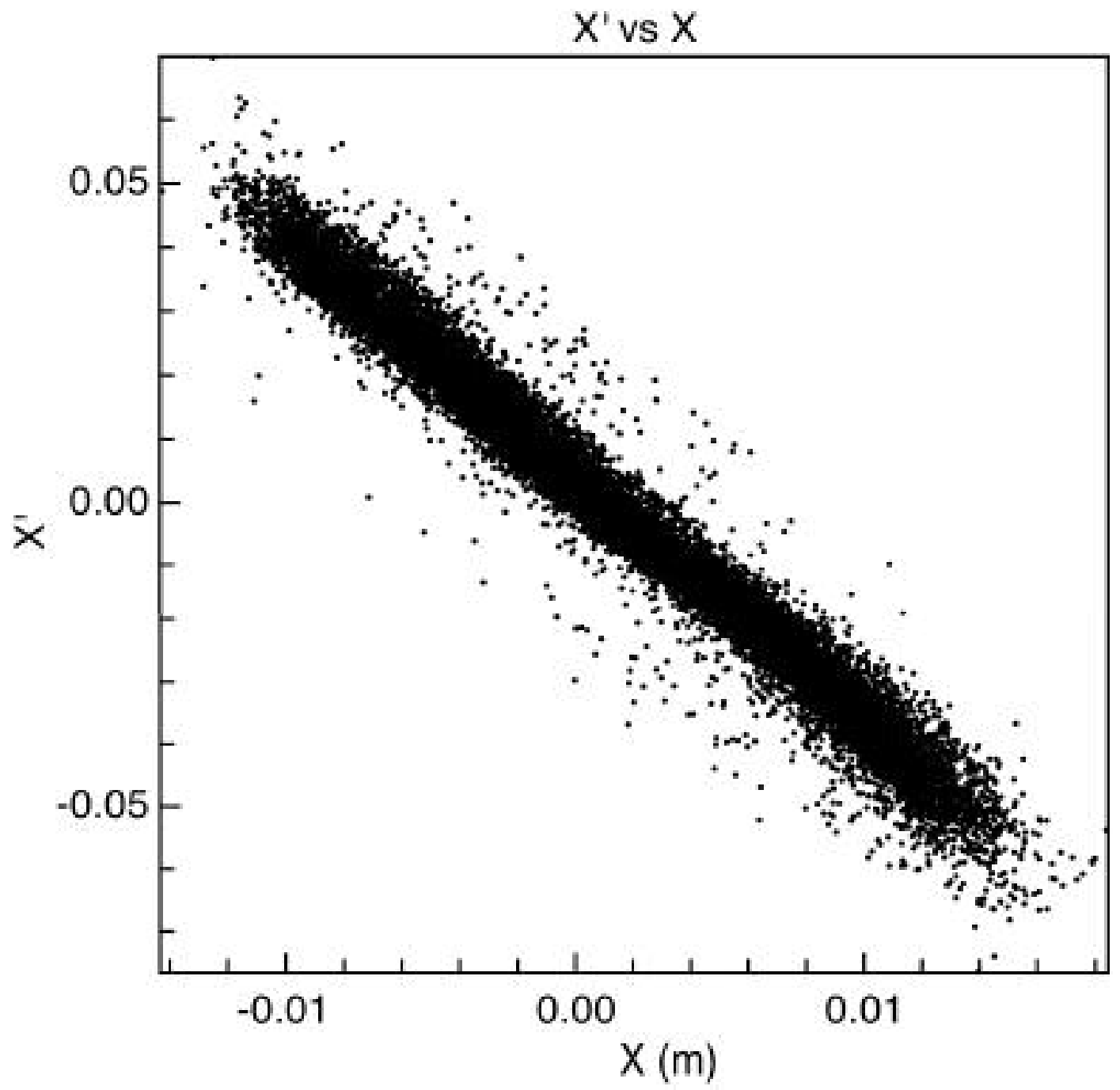

Figure 4: Plot of PIC simulation $\mathrm{x}-\mathrm{x}^{\prime}$ phase space at $17.1 \mathrm{~m}$ downstream from source, about $3 \mathrm{~m}$ after end of matching section. 


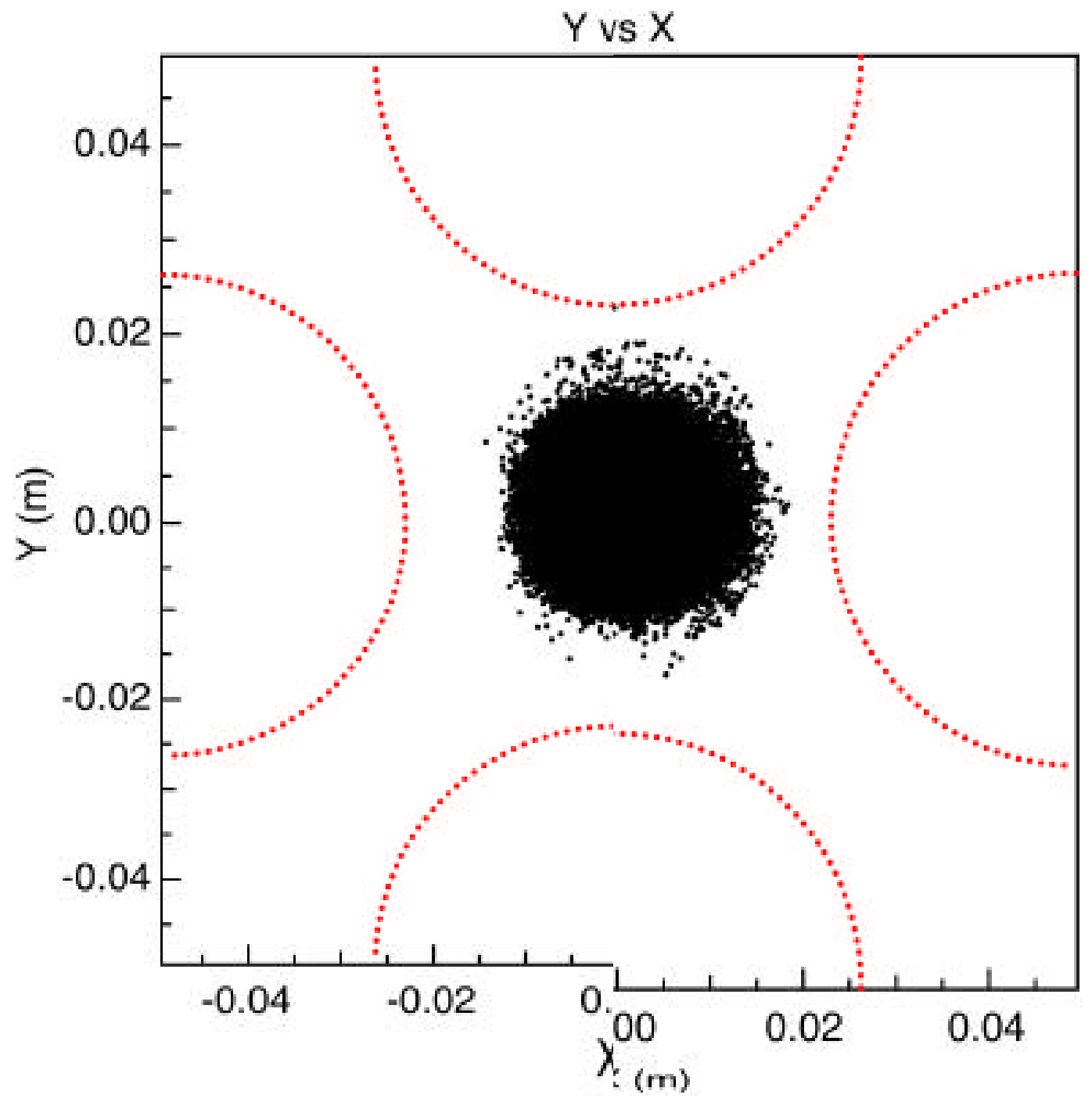

Figure 5: Plot of PIC simulation x-y phase space at $17.1 \mathrm{~m}$ downstream from source, about $3 \mathrm{~m}$ after end of matching section. 


\section{Tables}

\begin{tabular}{l|ccc}
\hline & Q5 & Q27 & Q53 \\
\hline$V_{q}(\mathrm{kV})$ & 243.9 & 163.2 & 130.9 \\
$L(\mathrm{~cm})$ & 35.30 & 19.10 & 13.46 \\
$b(\mathrm{~cm})$ & 7.989 & 3.580 & 2.305 \\
$d(\mathrm{~cm})$ & 4.497 & 1.668 & 0.085 \\
\hline
\end{tabular}

Table 1: List of quadrupoles parameters for

several quadrupoles in the 84 beam Adiabatic

Matching Section. 